\title{
Krüger, Jens Oliver; Roch, Anna; Breidenstein, Georg (2020) (Hrsg.): Szenarien der Grundschulwahl. Eine Untersuchung von Entscheidungsdiskursen am Übergang zum Primarbereich
}

\author{
Wiesbaden: Springer VS. 275 Seiten, ISBN: 978-3-658-22463-9, \\ 34,99€
}

\author{
Jennifer Gerlach \\ Online publiziert: 10 . August 2020 \\ (c) Der/die Autor(en) 2020
}

Dass die Einschulung eines Kindes von Eltern als Wahl oder Entscheidung begriffen wird, scheint mit Blick auf die Gesetzgebungen in Deutschland keinesfalls selbstverständlich. So bestehen in einem Großteil der Bundesländer sog. Einzugsgebietsregelungen, die - so könnte man meinen - die Frage auf welche Grundschule ein Kind gehen soll, in weiten Teilen vorbestimmen. Dass die Einschulung eines Kindes aber (zumindest von bestimmten Eltern) zunehmend als Entscheidung zwischen verschiedenen Schulen begriffen wird, macht die Forscher*innengruppe um Georg Breidenstein zum Ausgangspunkt des von der Deutschen Forschungsgesellschaft geförderten Projekts „Exzellenz im Primabereich. Die ,Beste Schule“ als Gegenstand der Aushandlung im Entscheidungsdiskurs der Eltern“, dessen Ergebnisse nun in dem Band „Szenarien der Grundschulwahl“ präsentiert werden. Die Forscher*innen fragen, wie es dazu kommt „dass die Einschulung des Kindes aktuell von vielen Eltern als Frage nach der besten oder passenden Schule gehandhabt und als Entscheidung begriffen wird“ und danach, ,wie (...) Entscheidungsoptionen und - Notwendigkeiten im Zusammenhang der Einschulung generiert" werden (Breidenstein, Krüger, Roch 2020, S. 6). Diesen Fragen gehen sie aus einer diskursanalytischen Perspektive nach.

Entsprechend der Konzeption der Grundschulwahl als eine Entscheidung, die nicht automatisch gegeben, sondern erst zu einer gemacht werden muss, wird eine performanztheoretische Perspektive auf Entscheidungen eingenommen. Diese Perspektive, die einleitend in einem Beitrag von Jens Oliver Krüger entwickelt wird, nimmt gegenüber anderen Theoretisierungen von Entscheidungen, die auf Resultate von Entscheidungen fokussieren, eine Blickumkehrung vor: Sie fragt nach der (dis-

\footnotetext{
J. Gerlach $(\bowtie)$

Arbeitsbereich Schulpädagogik/Empirische Unterrichtsforschung und Schulentwicklung,

Georg-August-Universität Göttingen, Göttingen, Deutschland

E-Mail: Jennifer.Gerlach@ sowi.uni-goettingen.de
} 
kursiven) Hervorbringung von Entscheidungsszenarien und markiert anstelle einer Fokussierung auf Berechenbarkeit und Entscheidbarkeit gerade Unentscheidbarkeit, Unberechenbarkeit und Unabgeschlossenheit von Entscheidungen als zentrale Momente in Entscheidungsprozessen.

Diese Perspektive erweist sich für die empirische Erforschung insofern als gewinnbringend, als dass sie mit ihrem Fokus auf die Hervorbringung von Entscheidungszenarien unterschiedliche Kontexte in den Blick zu nehmen vermag, in denen die Grundschulwahl als Entscheidung prozessiert und hervorgerbacht wird. Wie die Autor*innen selbst betonen, erlaubt die performanztheoretische Perspektivierung der Grundschulwahl also, die Schulwahl jenseits einer Fokussierung auf elterliches Wahlverhalten oder auf die Etablierung von wettbewerbsorientierten Steuerungsstrategien zu untersuchen und beiden Aspekten Berücksichtigung zu schenken (vgl. ebd. S. 11). Der vorliegende Band verdeutlicht den Ertrag einer solchen Perspektive auf die Grundschulwahl anhand von zahlreichen empirischen Einzelstudien. Wie dies im Detail gelingt und welche Sichtbarkeiten geschaffen werden, soll im Rahmen dieser Rezension exemplarisch anhand ausgewählter Aspekte von zwei Einzelstudien aus dem Band skizziert werden. Zunächst sei jedoch der Aufbau des Bandes dargestellt.

Der Band gliedert sich neben einem einführenden Teil, in dem in das Thema des Forschungsprojekts eingeführt wird und die performanztheoretische Perspektive entfaltet wird, in vier weitere Teile: Bildungsgerechtigkeit (Teil II), Grundschulqualität (Teil III), Elternverantwortung (Teil IV) und Epilog (V). Die Gliederung des empirischen Teils des Bandes (Teil II, III \& IV) erfolgt damit entlang von zentralen „Knotenpunkte[n]“ des Diskurses zur Grundschulwahl (ebd., S. 16). Jedes dieser empirischen Kapitel versammelt vier Einzelstudien, in denen auf Grundlage unterschiedlicher Materialsorten jeweils unterschiedliche Aspekte des Knotenpunktes in den Fokus gerückt werden. Der Blick auf die unterschiedlichen Materialsorten kann einen ersten Eindruck davon vermitteln, wie entlang dieser Konzeption der Blick für die Pluralität der Kontexte bzw. Szenarien geöffnet wird, in denen die Grundschulwahl als Entscheidung hervorgebracht und prozessiert wird: Gearbeitet wird mit Interviews (mit Eltern, Schulleitungen, einem Schulamtsleiter), ethnographischen Protokollen (von Tagen der offenen Tür, von Gesprächen und Situationen, in denen die Schulwahl thematisiert wird) und Dokumenten (Protokoll von einer Stadtratssitzung, auf der der Neuzuschnitt eines Schulbezirks diskutiert wird, Elternratgebern, Schulhomepages, einem Bogen mit dem elterliche Schulwünsche erfasst werden, Zeitungsberichten). Abgeschlossen wird der Band mit einem Epilog (IV) zu der Verstrickung der Schulwahlforschung in ihren Gegenstand.

Der erste hier exemplarisch vorgestellte Beitrag fragt auf Grundlage von Elterninterviews nach der praktischen Bearbeitung des Segregationsverdachts im Kontext der Grundschulwahl (Titel des Beitrags: Schulwahl als Elitebildung? Zur Bearbeitung des Segregationsverdachts im elterlichen Diskurs). Dabei kann gezeigt werden, dass Eltern den Zusammenhang von Schulwahlen und sozialer Segregation durchaus reflektieren und problematisieren. Insofern sich die Eltern selbst als ,wählende“ Eltern positionieren, stellt sie die Anerkennung des Zusammenhangs von Schulwahl und sozialer Entmischung vor Legitimationsprobleme (ebd., S. 52). Die Beschreibung der praktischen Bearbeitung dieser Legitimationsprobleme scheint nun insofern ergiebig, als dass erstens die Beschreibung einer ,soziale(n) Durchmischung“ an der 
gewählten Schule als Legitimationsfigur herausgearbeitet werden kann, die den Segregationsverdacht durch Schulwahl zu entkräften vermag (ebd., S. 47). Zweitens und das scheint zentral - kann mit Fokus auf die praktische Bearbeitung der Legitimationsprobleme die Eingebundenheit von Eltern in zwei Orientierungen aufgezeigt werden, in deren Spannungsverhältnis sich Eltern positionieren müssen: „Egalität und Chancengleichheit" und ,pädagogische Verantwortung für das Wohl des eigenen Kindes" (ebd., S. 53 Hervorh. im Orig.). Mit Fokus auf die Bearbeitung des Spannungsverhältnisses zwischen den o.g. Orientierungen wird sodann gezeigt, wie die diskursive Referenz auf Aspekte, die der zweiten Orientierung zugeschrieben werden können (bspw. „Bildungsinteresse“), es ermöglicht, die Abwendung von der Regelschule oder von bestimmten Regelschulen zu legitimieren und damit etwaige segregierende Effekte in Kauf zu nehmen. Die theoretische Sensibilisierung für Unentscheidbarkeit, Unabgeschlossenheit und Unberechenbarkeit vermag damit Forschungsbefunde, die Abgrenzungsbewegungen als elterliches Motiv der Grundschulwahl benennen (vgl. ebd., S. 45), nicht lediglich positiv zu verdoppeln, sondern zu differenzieren und die Eingebundenheit wählender Eltern in eine unentscheidbare Situation zwischen diesen Orientierungen aufzuzeigen.

Fokussiert dieser Beitrag auf die Elternperspektive, lässt sich der Gewinn einer performanztheoretischen Perspektive, die auf die Hervorbringung von Entscheidungszenarien fokussiert, in dem Band auch entlang instruktiver Verbindungen von Untersuchungsperspektiven verdeutlichten. Dies sei exemplarisch an einem weiteren Beitrag, der sich der Eigenlogik von Tagen der offenen Schultür widmet, veranschaulicht (Titel des Beitrags: Atmosphäre, Ambiente, Stimmung. Besuche an Tagen der offenen Schultür): Der Beitrag nimmt seinen Ausgangspunkt in der wiederholten Bezugnahme von Eltern in Interviews auf Atmosphären und Stimmungen als Argumente im Schulwahldiskurs. Ausgehend von der Annahme, dass für eine Aufnahme von Stimmungen und Atmosphären eine Präsenz an einem Ort erforderlich ist, die Eltern an Tagen der offenen Tür ermöglicht wird, befasst sich der Beitrag mit diesen Veranstaltungen. Tage der offenen Schultür finden in der Schulwahlforschung zwar bereits vereinzelt Berücksichtigung, doch erscheint die Konzeption des Beitrags insofern innovativ, als dass die Konstruktion des Gegenstands als Eigenlogik von Tagen der offenen Tür (bzw. das Interesse an Atmosphäre, Ambiente und Stimmung) es ermöglicht, die Tage der offenen Tür jenseits einer einseitigen Konzentrierung auf Inszenierungen der Institutionen oder auf Eindrücke der besuchenden Eltern zu fassen. So nähert sich der Beitrag seinem Gegenstand auf verschiedenen Wegen: Auf Grundlage von ethnographischen Protokollen und Literatur wird nach Herausforderungen gefragt, die sich mit der Gestaltung der Tage der offenen Schultür verbinden, und diese in Form von „Spannungsfeldern“ zu explizieren gesucht (ebd., S. 132). Zudem werden Variationen der Ausgestaltung der Tage der offenen Tür, anhand von ethnographischen Beobachtungsprotokollen expliziert und dabei Wahrnehmungen von Problemen der jeweiligen Variante benannt. Darüber hinaus wird sich mit den Wahrnehmungen von Tagen der offenen Tür, bzw. den „Spielräume(n) (...) sich zu dem, was dort wahrgenommen wird, in ein Verhältnis zu setzen“, befasst (ebd., S. 130).

Der Wert dieser Beschreibungen lässt sich nicht nur darin festmachen, dass sich dem Gegenstand aus verschiedenen Perspektiven (Institution, Besucher*in der Tage 
der offenen Tür) genähert wird, sondern dass mit einer Fokussierung auf „Spannungsfelder“ und „Spielräume“ innerhalb der verschiedenen Perspektiven, Strittigkeiten sichtbar gemacht - und auf die Pluralität der In-Verhältnis-Setzungen zu Tagen der offenen Tür fokussiert wird - also der Gegenstand in seiner Differenzialität sichtbar gemacht wird (ebd., S. 130, 132). Ausgehend von dem Nebeneinander der Perspektiven werden sodann Relationen hergestellt, in denen die Tage der offenen Schultür jenseits einer berechenbaren Planbarkeit in den Blick kommen. So wird formuliert: ,Während instiutionenseitig mit Inszenierungen von Tagen der offenen Tür an der Herstellung einer stimmungsvollen Atmosphäre gearbeitet wird, ist die Gestimmtheit auf Seiten der Besucher*innen davon abhängig, dass diese sich einer Stimmung überlassen“ (ebd., S. 140). Atmosphären und Stimmungen werden schließlich als eine ,Zwischenwelt zwischen konkreter Örtlichkeit, an der eine bestimmte Stimmung herrscht und einem Subjekt, das diese Stimmung empfindet", verortet (ebd., S. 140).

Können diese Ausführungen nur einen ersten Einblick in den Band darstellen, legen die Autor*innen eine Projektpublikation vor, deren Ertrag sich nicht allein in einem kreativen, mehrperspektivischen Beitrag zum Forschungsfeld der Grundschulwahl erschöpft, sondern mit dem bisherige Forschungsbefunde auch theoretisch erweitert und differenziert werden können. Mit der performanztheoretischen Perspektivierung der Frage nach Entscheidungen als Hervorbringung von Entscheidungsszenarien liegt zudem eine insofern vielversprechende Theoretisierung vor, als dass sie heuristisch weitere empirische Untersuchungen - nicht nur im Feld der Schulwahlen - anleiten könnte.

Funding Open Access funding provided by Projekt DEAL.

Open Access Dieser Artikel wird unter der Creative Commons Namensnennung 4.0 International Lizenz veröffentlicht, welche die Nutzung, Vervielfältigung, Bearbeitung, Verbreitung und Wiedergabe in jeglichem Medium und Format erlaubt, sofern Sie den/die ursprünglichen Autor(en) und die Quelle ordnungsgemäß nennen, einen Link zur Creative Commons Lizenz beifügen und angeben, ob Änderungen vorgenommen wurden.

Die in diesem Artikel enthaltenen Bilder und sonstiges Drittmaterial unterliegen ebenfalls der genannten Creative Commons Lizenz, sofern sich aus der Abbildungslegende nichts anderes ergibt. Sofern das betreffende Material nicht unter der genannten Creative Commons Lizenz steht und die betreffende Handlung nicht nach gesetzlichen Vorschriften erlaubt ist, ist für die oben aufgeführten Weiterverwendungen des Materials die Einwilligung des jeweiligen Rechteinhabers einzuholen.

Weitere Details zur Lizenz entnehmen Sie bitte der Lizenzinformation auf http://creativecommons.org/ licenses/by/4.0/deed.de. 